\title{
A NOVEL AUTOMATIC CLUSTER TRACKING ALGORITHM
}

\author{
Nicolai Czink, Christoph Mecklenbräuker \\ Forschungszentrum Telekommunikation Wien (ftw.) \\ Vienna, Austria \\ $\{$ czink,cfm\}@ftw.at
}

\begin{abstract}
On the way to answer the controversial question "What is a cluster?", we introduce a novel cluster tracking mechanism which is based on the multi-path component distance (MCD). We perform a temporal tracking of cluster centroids in the multidimensional parameter domain, starting from cluster estimates obtained by a recently introduced framework which automatically clusters parametric MIMO channel data.

To validate our algorithm, we use both synthetic and measured MIMO channels. We generate the synthetic channel using the IlmProp simulation tool. For the real-world channels we use data from an outdoor measurement campaign in a rural area.

Simulation results with synthetic channels validate the tracking algorithm, whereas its application on measurement data shows the occurrence of several clusters with different lifetimes.
\end{abstract}

\section{INTRODUCTION}

The problem of identifying clusters is currently the perhaps most controversial topic in channel characterisation. Discussions start right at the definition of a cluster itself. The only common agreement seems to be that a cluster should show some kind of similar parameters, which evolve smoothly over time.

In many papers visual inspection of measurement data from single snapshots was used [1, 2], which becomes impractical for large amounts of measurement data. Recently, an automatic algorithm was introduced in [3], which is based on clustering windowed parametric estimates and tracking the cluster centroids. The window-based clustering algorithm was subsequently improved by using the multi-path component distance (MCD) as the distance function in [4]. This algorithm was further improved by including the paths' power into the cluster identification and extended to a whole framework. The framework clusters the environment, decides on the correct number of clusters and prunes outliers. This is done for every single data window [5].

We also adopt the definition of "cluster" from this paper:

For a given number of clusters, clusters are chosen such that they minimize the total distance from their centroids. This implies that the cluster angular and cluster delay spreads are minimized.

This work was conducted within the EC funded network of excellence NEWCOM. We thank Elektrobit Testing Ltd. for providing their parameter estimator.

\author{
Giovanni Del Galdo \\ Communications Research Lab, University of Ilmenau \\ Ilmenau, Germany \\ gdg@tu-ilmenau.de
}

The last step is to subsequently track the obtained clusters. By cluster tracking we are able to extract the timeevolution of the parameters. The algorithm is validated by tracking MIMO channel parameters of a synthetic timevariant environment generated by the IlmProp channel model [6].

\section{PROBLEM}

The starting point is a large number of paths estimated from time-varying multi-dimensional parametric MIMO channel data. Each path is characterized by its delay, angles-ofarrival (AoA) and angles-of-departure (AoD). It has been investigated in several studies that these parameters tend to appear in clusters, i.e. in groups of multi-path components (MPCs) with similar angles and delays. The problem is to find an automatic procedure to identify and track these clusters.

For cluster identification, we use a novel framework introduced recently [5, 7], which clusters the environment, decides on the correct number of clusters, and prunes outliers. The input data to the clustering algorithm is an $K \times L$ matrix, where $K$ is the number of estimated multipaths, and $L$ is the number of channel parameters. Typically, the dimensions of $L$ are power $(P)$, delay $(\tau)$, azimuth and elevation $\mathrm{AoA}\left(\varphi_{\mathrm{AoA}}, \theta_{\mathrm{AoA}}\right)$ and azimuth and elevation $\mathrm{AoD}$ $\left(\varphi_{\mathrm{AoD}}, \theta_{\mathrm{AoD}}\right)$. The output of the clustering algorithm is a set of cluster centroids in parameter space together with the allocated MPCs for each clustered scenario. In this paper we neglect the elevation domains, as clusters are insufficiently separated there.

To identify the time-variant behaviour of clusters, we need to track the cluster centroids over several time windows. The tracking algorithm must be able to decide on cluster birth, death, or movement.

\section{TRACKING ALGORITHM}

The cluster tracking mechanism is able to capture the movement of clusters with very low complexity. The idea is based on the distance between the clusters' centroids. As the centroids are given in the multi-dimensional parameter space, i.e. angles and delay, we chose the MCD [8] as suitable distance metric to cope with angular periodicity as well as data scaling.

Two subsequent sets of a number of $N_{\text {old }}$ old and $N_{\text {new }}$ new cluster centroids $\mathbf{c}_{i}^{\text {(old) }}$ and $\mathbf{c}_{j}^{\text {(new) }}$, are considered, where $i=1 \ldots N_{\text {old }}$ and $j=1 \ldots N_{\text {new }}$. The algorithm is given in Alg 1. In the following we describe the parts of the algorithm. 


\section{Tracking algorithm:}

1. Calculate the distance between any old and any new centroid using the MCD.

2. For each new centroid:

a. Calculate the distance and index of the closest old centroid.

b. IF smallest distance $>$ threshold, treat centroid as new cluster

3. For each old centroid:

a. Check number of close new centroids within distance threshold

b. IF number $=1$, old cluster moved.

c. IF number $>1$, cluster split:

- closest new cluster is treated as old cluster moved

- other close ones are treated as new clusters

Alg. 1: Tracking Algorithm

Ad 1) The distances between the centroids are arranged in the distance matrix $\mathbf{D}$ with dimension $N_{\text {old }} \times N_{\text {new }}$, where each element is calcualted as

$$
[\mathbf{D}]_{i j}=\operatorname{MCD}\left(\mathbf{c}_{i}^{\text {(old) }}, \mathbf{c}_{j}^{\text {(new) }}\right),
$$

i.e. the distance between the $i$ th old and $j$ th new centroid. All further evaluations can now easily be done by searching in the distance matrix.

Ad 2a) For each column of $\mathbf{D}$, search for the smallest entry in the distance matrix. The indices $i^{*} j^{*}$ of this value identifies the closest old cluster.

Ad 2b) If the distance $[\mathbf{D}]_{i^{*} j^{*}}$ between a new cluster and the closest old cluster exceeds a specified threshold $\varepsilon$, the cluster is treated as a new cluster.

Ad 3) We now check for each old cluster, if it has moved.

Ad 3a) For each row in $\mathbf{D}$ count the number of elements smaller than $\varepsilon$.

Ad 3b) If only one new cluster is in the vicinity of the old cluster, the old cluster has moved.

Ad 3c) If many new clusters are in the vicinity of the old cluster, the old cluster moved towards the closest new one. The other close ones are treated as new.

To every new cluster a unique cluster-ID (CLID) is assigned. If a movement is identified, the moved cluster inherits the CLID from its predecessor.

\section{Scenarios}

We used both synthetic and measured channels to validate our tracking algorithm.

\section{A. Synthetic validation scenario}

To validate the clustering framework and the tracking algorithm, we use a synthetic time-variant scenario obtained by the IlmProp channel model [6]. The IlmProp is a flexible geometry-based Multi-User MIMO channel modelling tool, capable of dealing with time variant frequency selective scenarios. The BS and mobile terminals can employ any number of antennas arranged in an array with an arbitrary geometry. The channel is computed as a sum of the Line Of Sight (LOS) and of a number of rays which represent the multi-path components. The latter are obtained by point-like scatterers, which can be placed at will. The model supports both single- and multiple-reflections. The information about where the scatterers are, and how the paths are linked to them can be set arbitrarily, or it can be derived by parameter estimations from channel measurements. The information about the scenario is stored in form of Cartesian coordinates and their evolution in time. The scenario may include obstacles (such as buildings), which can obstruct the propagation paths.

Figure 1 shows the scenario we use to validate the automatic cluster tracking algorithm. A single mobile (MS) is moving on a linear trajectory (along the $x$-axis) towards the Base Station (BS). The LOS path was artificially suppressed. We consider the uplink phase, so that the BS acts as the receiver.

The scenario exhibits 8 separate clusters. The cluster numbering corresponds to the automatic cluster identification (see Section V.). All propagations paths are characterized by single bounce reflections with the exception of the ones named $6 a$ and $6 b$, which realize double bounce paths. Solid black lines indicate the interconnections between MS, clusters and the BS. The path towards the cluster number 10 is obstructed by a building during most of the mobile's trajectory. For this reason it is marked by a dashed line.

The clustering algorithm is meant to identify clusters from the geometrical parameters extracted from channel measurements by a parameter estimation technique. The idea is to extract these parameters, i.e. delay time, direction of arrival and departure, and path-strength, directly from the IlmProp, and feed them to the cluster identification framework. Subsequently, the clusters are tracked. In the next section we consider the issue of the variance introduced by the parameter estimator, showing that for the aims of our investigation it can be neglected.

\section{B. Measured environment}

In this paper we use data from a rural outdoor MIMO measurement campaign [9] ${ }^{1}$. The center frequency was $2 \mathrm{GHz}$ with a bandwidth of $120 \mathrm{MHz}$.

The measurements were performed by the MIMO capable wideband vector channel sounder RUSK-ATM [10]. The sounder was specifically adapted to operate at a center frequency of $2 \mathrm{GHz}$ with an output power of 2 Watt. The transmitted signal is generated in frequency domain to ensure a pre-defined spectrum over $120 \mathrm{MHz}$ bandwidth, and approximately a constant envelope over time. At the

\footnotetext{
${ }^{1}$ Selected data sets from urban, suburban, and indoor environments are available for download at http://measurements. ftw. at/.
} 


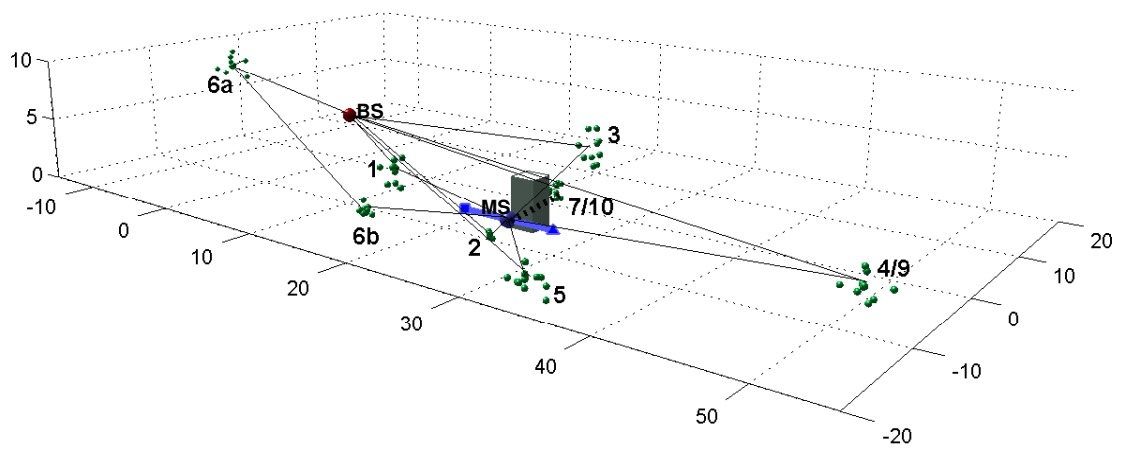

Figure 1: Scenario used to validate the automatic cluster tracking algorithm; cluster numbers correspond to the output of the tracking algorithm

receiver the input signal is correlated with the transmitted pulse-shape in the frequency domain resulting in the specific transfer functions. Back-to-back calibration before each measurement ensured an unbiased estimate. Furthermore, transmitter and receiver were synchronized via rubidium clocks at either end for accurate frequency synchronism and a defined time-reference. For studies on MIMO systems, the double-directional nature of the channel must be exploited. Therefore two simultaneously multiplexed antenna arrays have been used at transmitter and receiver.

At the transmitter a uniform circular array consisting of 15 monopoles mounted on a trolley was used. The receiver was connected to an 8-element uniform linear patch array. Two dummy elements on each side of the array ensure equal element characteristics for all elements. For the measurements the transmitter was placed on a trolley and moved through streets at speeds of about 3 to $6 \mathrm{~km} / \mathrm{h}$.

We chose a suburban scenario, the village of Weikendorf. The measurement area covers one-family houses with private gardens around them. The houses are typically one floor high. Such an area is common for small villages around Vienna. In addition there is also a rail track in this area which breaks the structure of single placed houses. The receiver was at a height of about $20 \mathrm{~m}$ which is much higher than anything in the surroundings. Therefore the transmitter operated ofter in the LOS regime. The most interesting measurement run within this area includes a walk through a small pedestrian tunnel below the railway. A photograph of the scenario is given in Figure 2. We use 485 equidistant snapshots of the MIMO channel, which are spaced $0.26 \mathrm{~m}$ corresponding to 1.7 wavelengths.

\section{RESUlts}

We assessed the performance of this cluster tracking algorithm with both synthetic and real-world measurement data.

\section{A. Simulation results}

Figure 1 shows the simulated scenario, details are provided in Section IV. The channel model provided 80 subsequent equidistant snapshots in time of the smoothly time-varying channel, while the MS moved towards the BS.

For clustering the synthetic scenario, we collected 3 subsequent time snapshots in a sliding window. The clustering

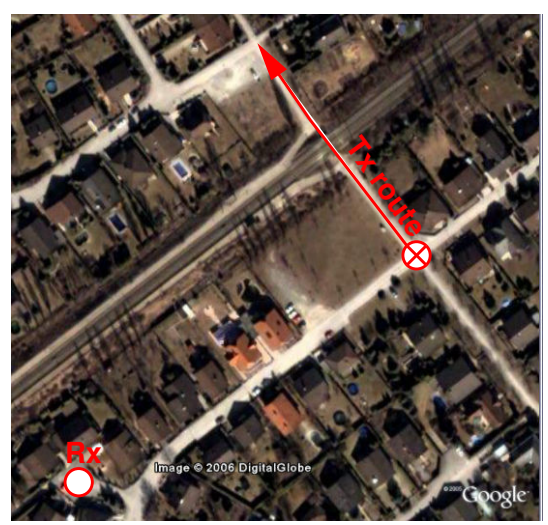

Figure 2: Map of measurement environment (Copyright: Google Earth, http: / / earth.google.com/)

algorithm identified between 6 and 7 clusters in each time window. Subsequently, the tracking algorithm was applied.

The results are shown in Figure 3, where the evolution of the clusters over time are shown in ascending order (a)-(f). Clusters are indicated by coloured spheres, paths are shown as crosses in the spheres. The number next to the sphere indicates the unique CLID.

Figure 3 a shows the first time window, where seven clusters were identified. Clusters 1 and 2 are quite close to each other, but can still be clearly distinguished by the algorithm. Cluster 7 describes the propagation paths, which will be shadowed in the following windows. In the next time windows (b)-(d) cluster 4 is split into 2 clusters, because some of the components in cluster 4 are quite separated. The missing cluster 8 was also a short-living appearance of cluster 9. Moreover, all clusters move in the parameter space, most prominently, clusters 1 and 2 start to separate. In the last time windows (e)-(f) the previously shadowed cluster appears again and gets a new CLID 10. Both, the identification and tracking were achieved fully automatic without any user interaction.

\section{B. Results from measurements}

We estimated propagation paths out of the impulse responses using the ISIS (Initialization-and-Search-Improved SAGE) algorithm [11] and subsequently clustered them using the automatic clustering algorithm presented in [5]. The 


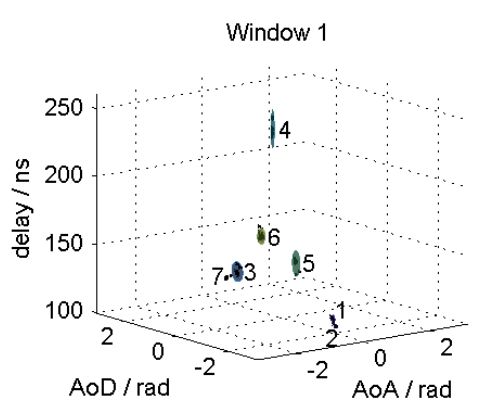

(a)

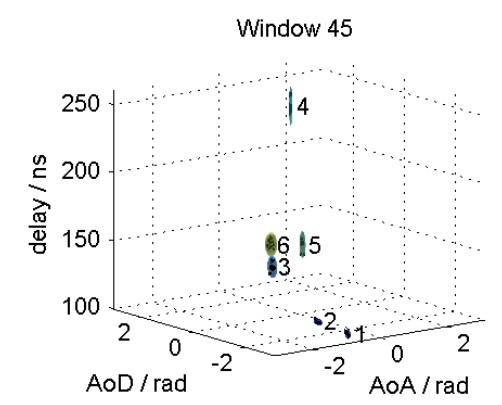

(d)

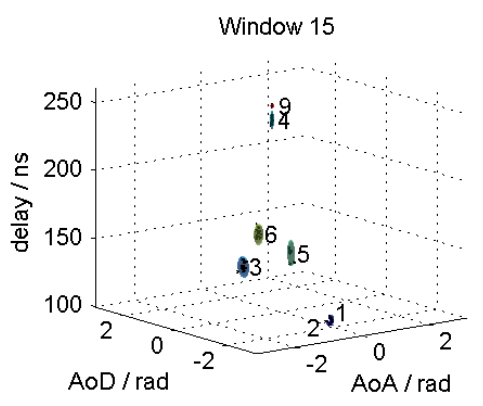

(b)

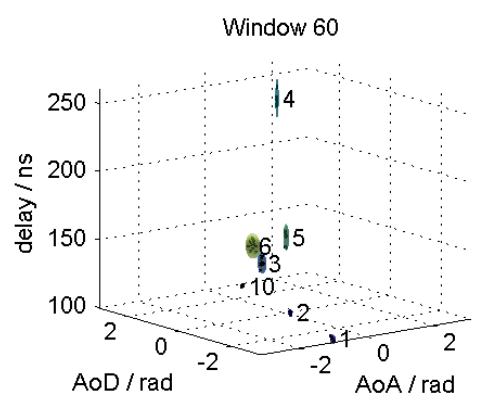

(e)

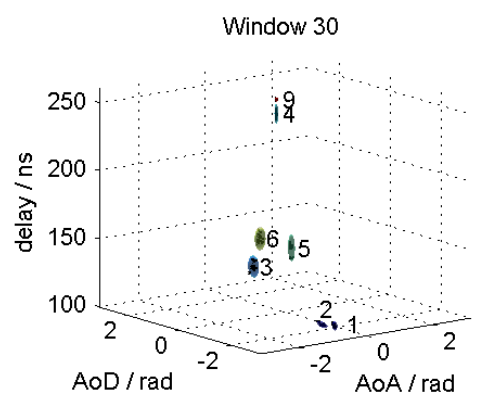

(c)

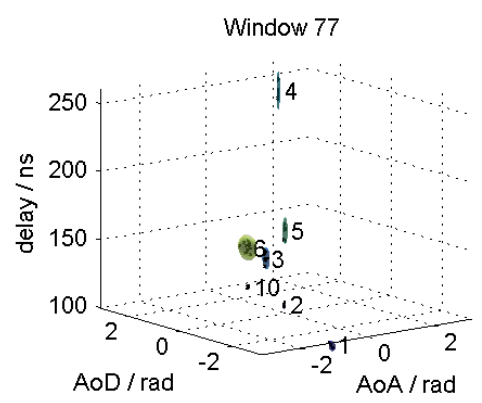

(f)

Figure 3: Results from cluster tracking performed on the synthetic scenario shown in Figure 1: (a)-(f) represent different time windows in ascending order

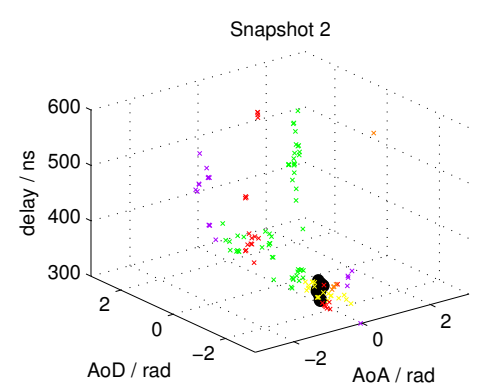

(a)

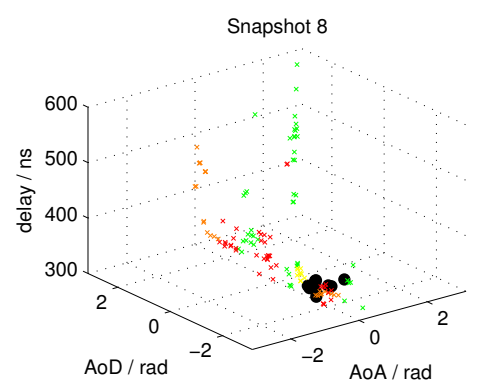

(d)

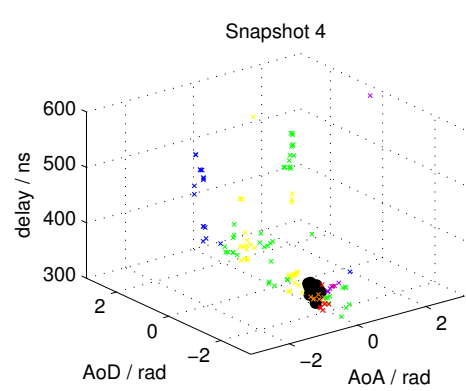

(b)

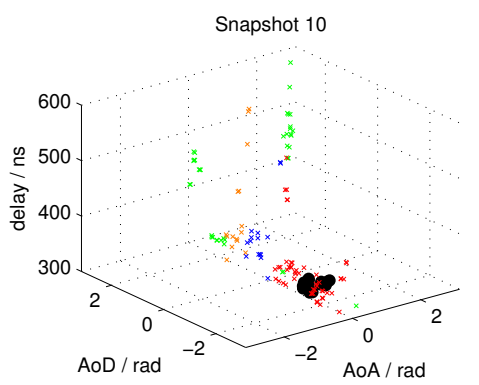

(e)

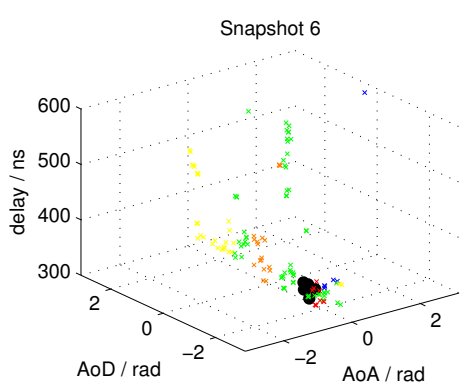

(c)

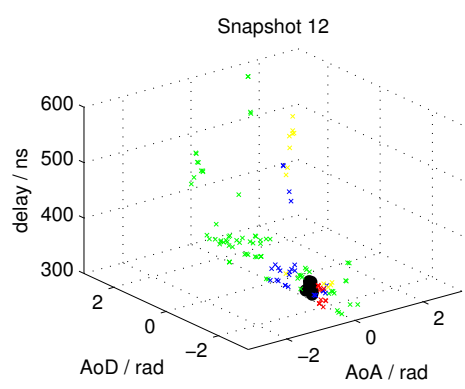

(f)

Figure 4: Results from tracking measurement data: (a)-(f) represent different time windows in ascending order. Paths from the tracked cluster (CLID = 366) are indicated by black circles, other paths are indicated by crosses colour-coded by their CLID. 


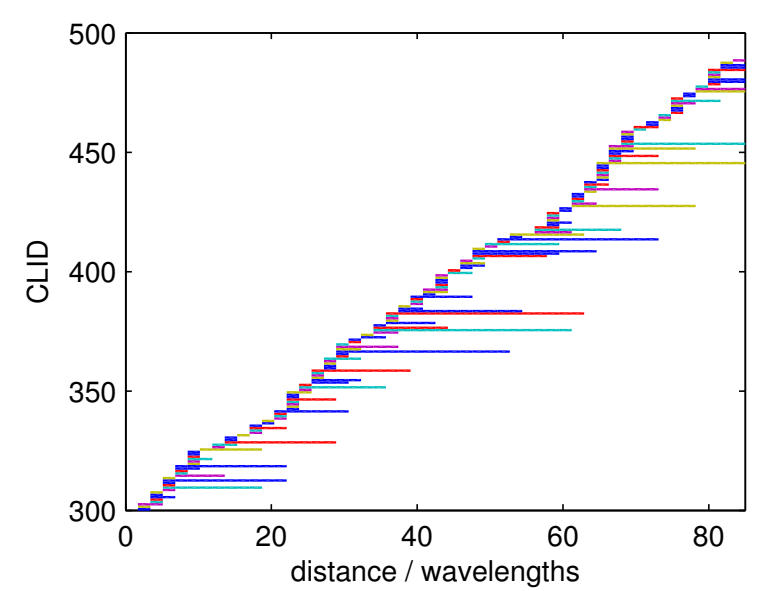

Figure 5: Cluster lifetimes for a selected time window

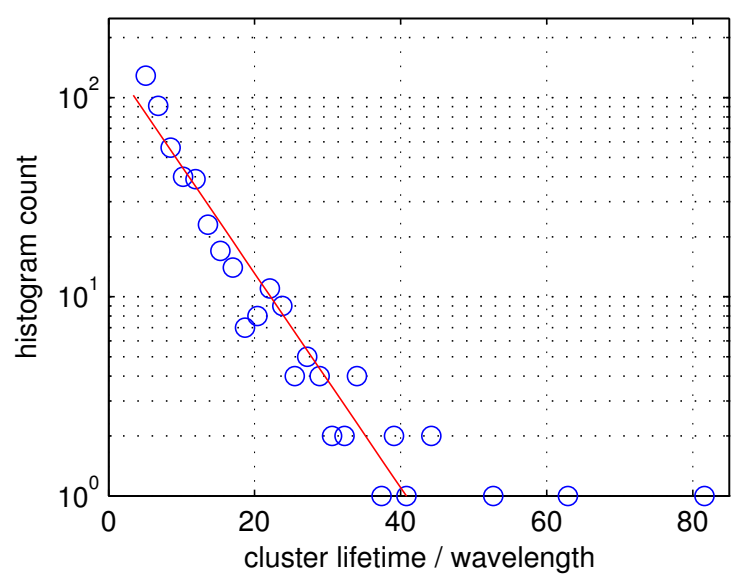

Figure 6: Histogram of cluster lifetimes over all snapshots

cluster centroids were tracked using the presented algorithm with a tracking range of $\varepsilon=0.5$.

Figure 5 shows the results for a window of 50 selected consecutive snapshots where the clusters were tracked. We plotted the snapshot number (abscissa) vs. the CLID (ordinate). The lengths of the horizontal lines indicate the lifetime of the respective clusters.

We observed that many clusters exist for only one snapshot and could not be tracked. Furthermore, there were outlier clusters with lifetimes larger than 50 wavelengths. We follow the strategy of truncating these clusters from our evaluations, as we cannot be sure if these clusters really exist or are just artefacts of the algorithm.

We provide a histogram of the observed cluster lifetimes for this scenario in Figure 6. The (truncated) mean cluster lifetime was found to be 7.9 wavelengths (corresponding to $1.2 \mathrm{~m}$ distance $)^{2}$.

To visualize the tracking results in the parameter space, we selected one demonstrative cluster and plot its evolution over time (see Figure $4 \mathrm{a}-\mathrm{f}$ ). One can observe that the cluster parameters change slightly over time, but the cluster centroid seems to be constant.

\footnotetext{
${ }^{2}$ In our idealised cluster model, clusters never re-appear after they have vanished: When a cluster vanishes, it's lifetime ends.
}

\section{CONCLUSIONS}

We demonstrated the performance of a novel cluster identification and tracking algorithm by clustering both synthetic and measured environments.

The synthetic environment was obtained from the IlmProp channel model where we were able to validate the performance of the tracking algorithm. The algorithm was able to correctly identify clusters and track their movement in the parameter space.

For tracking clusters from measurements, we used realworld data from an outdoor MIMO measurement campaign in a rural area. We found that the algorithm identifies many clusters which exist just for one snapshot. For our evaluations, we excluded them as we cannot be sure if they are true or an artefact of the algorithm. In this scenario we find a mean cluster lifetime of 4.9 snapshots.

\section{REFERENCES}

[1] K. Yu, Q. Li, D. Cheung, and C. Prettie, "On the tap and cluster angular spreads of indoor WLAN channels," in Proceedings of IEEE Vehicular Technology Conference Spring 2004, Milano, Italy, May 17-19, 2004.

[2] C.-C. Chong, C.-M. Tan, D. Laurenson, S. McLaughlin, M. Beach, and A. Nix, "A new statistical wideband spatio-temporal channel model for 5-GHz band WLAN systems," IEEE Journal on Selected Areas in Communications, vol. 21, no. 2, pp. 139 - 150, Feb. 2003.

[3] J. Salo, J. Salmi, N. Czink, and P. Vainikainen, "Automatic clustering of nonstationary MIMO channel parameter estimates,' in ICT'05, Cape Town, South Africa, May 2005, Cape Town, South Africa.

[4] N. Czink, P. Cera, J. Salo, E. Bonek, J.-P. Nuutinen, and J. Ylitalo, "Automatic clustering of MIMO channel parameters using the multipath component distance measure," in WPMC'05, Aalborg, Denmark, Sept. 2005.

[5] N. Czink and P. Cera, "A novel framework for clustering parametric MIMO channel data including MPC powers," in COST 273 Post-Project Meeting, Lisbon, Portugal, Nov. 2005, available at http://www.nt.tuwien.ac.at/staff/nicolai-czink/.

[6] G. Del Galdo, M. Haardt, and C. Schneider, "Geometry-based channel modelling of MIMO channels in comparison with channel sounder measurements," Advances in Radio Science - Kleinheubacher Berichte, pp. 117-126, October 2003, more information on the model, as well as the source code and some exemplary scenarios can be found at http://tu-ilmenau.de/ilmprop.

[7] N. Czink, P. Cera, J. Salo, E. Bonek, J.-P. Nuutinen, and J. Ylitalo, "A framework for automatic clustering of parametric MIMO channel data including path powers," 2006, submitted to IEEE Vehicular Technology Conference 2006 Fall.

[8] M. Steinbauer, H. Özcelik, H. Hofstetter, C. Mecklenbräuker, and E. Bonek, "How to quantify multipath separation," IEICE Trans. Electron., vol. E85, no. 3, pp. 552-557, March 2002.

[9] H. Hofstetter, C. Mecklenbräuker, R. Müller, H. Anegg, H. Kunczier, E. Bonek, I. Viering, and A. Molisch, "Description of wireless MIMO measurements at $2 \mathrm{GHz}$ in selected environments," in COST273 Temporary Document TD(02)135, Lisbon, Portugal, September 2002.

[10] R. Thomä, D. Hampicke, A. Richter, G. Sommerkorn, A. Schneider, U. Trautwein, and W. Wirnitzer, "Identification of time-variant directional mobile radio channels," IEEE Transactions on Instrumentation and Measurement, vol. 49, pp. 357-364, April 2000.

[11] B. H. Fleury, X. Yin, K. G. Rohbrandt, P. Jourdan, and A. Stucki, "Performance of a high-resolution scheme for joint estimation of delay and bidirection dispersion in the radio channel," Proc. IEEE Vehicular Technology Conference, VTC 2002 Spring, vol. 1, pp. 522526, may 2002. 